\title{
LA RENDICIÓN DE CUENTAS EN ESTABLECIMIENTOS EDUCATIVOS DE GUATEMALA
}

\author{
Patricia Luz Mazariegos Romero \\ Fermín Ferriol Sánchez \\ Universidad Internacional Iberoamericana (UNINI) (México)
}

\begin{abstract}
Resumen. La rendición de cuentas en el sistema educativo es el proceso que permite cumplir responsabilidades y alcanzar los objetivos propuestos, este es un tema que ha cobrado importancia al ser utilizada como una herramienta para fomentar la transparencia en el manejo de recursos, en ese contexto este estudio se realizó con el objetivo de revisar el sistema de indicadores educativos para implementar la rendición de cuentas en establecimientos educativos de Guatemala. Fue un estudio de alcance descriptivo, tomando como fuentes de información cinco profesionales expertos en educación, ochenta y nueve directores, doscientos veinticuatro docentes de escuelas públicas y diez técnicos expertos en acompañamiento pedagógico. Para recopilar información se aplicaron a dichos sujetos encuestas, se hicieron entrevistas y grupos focales. Los resultados permitieron establecer la situación del sistema de indicadores educativos implementado por el Ministerio de Educación y plantear una propuesta teórica que incluye la integración de una dimensión de indicadores de rendición de cuentas, como herramienta para fomentar la transparencia. El diseño de un sistema de indicadores que integre la dimensión de rendición de cuentas influirá positivamente en la gestión administrativa de establecimientos públicos; con esto se tendrán mayores posibilidades de encontrar establecimientos con mejores prácticas de accountability, ya que se ha evidenciado de forma empírica que la integración de la comunidad educativa de forma organizada genera mejores resultados de desempeño.
\end{abstract}

Palabras clave: Rendición de cuentas, indicadores educativos, rendición de cuentas, comunidad educativa.

\section{ACCOUNTABILITY IN EDUCATIONAL ESTABLISHMENTS IN GUATEMALA}

\begin{abstract}
Accountability in the education system is the process that allows to fulfill responsibilities and achieve the proposed objectives, this is an issue that has become important to be used as a tool to promote transparency in the management of resources, in this context this study was conducted with the aim of reviewing the system of educational indicators to implement the rendering of accounts in educational establishments in Guatemala. It was a study of descriptive scope, taking as sources of information five professional experts in education, eighty-nine directors, two hundred and twenty-four public school teachers and ten expert technicians in pedagogical support. To collect information, these subjects were applied to surveys, interviews and focus groups were conducted. The results allowed to establish the situation of the system of educational indicators implemented by the Ministry of Education and to propose a theoretical proposal that includes the integration of a dimension of accountability indicators, as a tool to promote transparency. The design of a system of indicators that integrate the accountability dimension will positively influence the administrative management of public establishments; With this, there will be greater possibilities of finding establishments with better accountability practices, since it has been evidenced empirically that the integration of the educational community in an organized manner generates better performance results.
\end{abstract}

Keywords: Accountability, educational indicators, educational community. 


\title{
RESPONSABILIDADE EM ESTABELECIMENTOS EDUCACIONAIS NA GUATEMALA
}

\begin{abstract}
Resumo. A responsabilização no sistema de ensino é o processo que permite cumprir as responsabilidades e alcançar os objetivos propostos, esta é uma questão que se tornou importante para ser usada como uma ferramenta para promover a transparência na gestão de recursos, neste contexto, este estudo foi conduzido com o objetivo de rever o sistema de indicadores educacionais para implementar a prestação de contas em estabelecimentos de ensino na Guatemala. Foi um estudo de escopo descritivo, tendo como fontes de informação cinco especialistas profissionais em educação, oitenta e nove diretores, duzentos e vinte e quatro professores de escolas públicas e dez técnicos especializados em apoio pedagógico. Para coletar informações, esses assuntos foram aplicados em pesquisas, entrevistas e grupos focais foram realizados. Os resultados permitiram estabelecer a situação do sistema de indicadores educacionais implementados pelo Ministério da Educação e propor uma proposta teórica que inclua a integração de uma dimensão dos indicadores de responsabilização, como ferramenta para promover a transparência. A concepção de um sistema de indicadores que integram a dimensão da responsabilidade influenciará positivamente a gestão administrativa dos estabelecimentos públicos; com isso, haverá maiores possibilidades de encontrar estabelecimentos com melhores práticas de prestação de contas, uma vez que foi evidenciado empiricamente que a integração da comunidade educacional de forma organizada gera melhores resultados de desempenho.
\end{abstract}

Palavras-chave: Responsabilidade, indicadores educacionais, comunidade educacional.

\section{Introducción}

Los esfuerzos por garantizar la calidad de la educación y de la gestión de las instituciones educativas requieren la implantación de una cultura de rendición de cuentas y el cumplimiento de indicadores educativos. Las escuelas, sus miembros y líderes tienen que responder a las necesidades de la sociedad en cuanto a calidad y al fomento de la transparencia.

Para mejorar la calidad educativa en varios países se están implementando estrategias, las cuales presentan el uso de indicadores educativos, según Martínez (2010) para evaluar los avances en este tema existe un macrosistema, un mesosistema y un micro sistema. El macrosistema incluye a) gestión y administración, b) normas y regulaciones, c) planes y programas. El mesosistema integrado por el centro educativo y el aula escolar. El microsistema integrado por alumnos, docentes y directores.

El indicador educativo es una medida estadística sobre aspectos que se consideran importantes de los sistemas educativos. Los indicadores resultan de la comparación de diferentes medidas estadísticas (Morduchowicz, 2006). Morduchowicz indica que la Organización de las Naciones Unidas para la Educación, las Ciencias y la Cultura (UNESCO) fue la pionera en el establecimiento de indicadores educativos, para conocer la eficiencia, calidad y equidad de un sistema educativo, fueron los que iniciaron las comparaciones internacionales.

Los indicadores son parámetros utilizados para medir el nivel de cumplimiento de una actividad o un evento, según indican García, Raez, Castro, Vivar \& Oyola ( (2003) estos generan información para la toma de decisiones, además permiten establecer el nivel de eficacia y eficiencia de una institución y la evaluación de programas. Los indicadores deben contar con nombre, forma de medición y unidad de medida. 
Utilizar un solo indicador para realizar análisis es insuficiente para tener información robusta y confiable, especialmente cuando se trata de procesos de alto grado de complejidad como lo son los procesos educativos. Morduchowicz (2006) indica que los indicadores no son cifras aisladas, estos se encuentran relacionados con otros indicadores y que deben agruparse y constituirse en sistemas de indicadores para lograr que su lectura y análisis sea de fácil comprensión y además brindar información sobre el componente de un sistema o del sistema completo, mostrando como se relacionan para producir diferentes efectos.

En el año 2013 el Ministerio de Educación de Guatemala con el apoyo de la Agencia Internacional para el desarrollo (USAID) presentó el sistema de indicadores, realizando algunas adecuaciones al que venían utilizando, este incluye cinco dimensiones adaptadas del modelo de calidad educativa que propuso la UNESCO en el 2005. Estas son las que se listan: 1. Contexto de la educación; 2. Recursos; 3. Procesos educativos; 4. Resultados de escolarización y eficiencia interna; 5. Resultados de aprendizaje del sistema.

Este sistema de indicadores se implementó con la finalidad de apoyar la rendición de cuentas agregando a este los resultados de las evaluaciones estandarizadas que se aplican de forma muestral a establecimientos educativos de primaria, básico y diversificado. Actualmente, se asocia a la rendición de cuentas con la evaluación de los aprendizajes y el monitoreo de los establecimientos educativos. El propósito fundamental de la evaluación educativa es la determinación empírica de la calidad. Señalan los autores que las funciones principales de la evaluación son: 1) la certificación y acreditación; 2) la rendición de cuentas; y 3) el aprendizaje de la organización (Scheerens, Glass, \& Thomas, 2007).

Según McMeekin y Di Gropello (2006), hay cuatro actores principales en el proceso de rendición de cuentas, siendo estos lo siguientes: 1) Usuarios del servicio educativo; 2) diseñadores de las políticas educativas, 3) proveedores de los servicios educativos, personal administrativo, autoridades locales, 4) proveedores directos del servicio educativo, personal docente. Por tanto, para buscar efectividad en estos procesos la mejor opción es presentarlo como un sistema participativo que integre a los actores educativos docentes, estudiantes, padres de familia y autoridades.

El enfoque de sistemas surge por la necesidad de unificar muchos campos del conocimiento, de buscar la interrelación e interdependencia de los procesos que produzcan los resultados sinérgicos eficaces. Este enfoque ha sido usado por las ciencias físicas, biológicas y sociales, como marco de referencia para la integración de la teoría organizacional moderna. Morales (2016) menciona que un sistema es aquel conjunto donde todos sus componentes están relacionados unos con otros y esas relaciones posibilitan su correcto funcionamiento. El cambio en uno o en una relación entre componentes, trae consecuencia en otros y en el funcionamiento general.

Arnold y Rodríguez (1999) definen sistema como una forma sistemática y científica de aproximación y representación de la realidad, también como una práctica estimulante para formas de trabajo transdisciplinarias. Lo importante para ellos son las relaciones y conjuntos que emergen. Por medio de las relaciones se dan interacciones entre especialistas y especialidades con una buena forma de comunicación.

Los autores mencionados presentan los objetivos originales de la Teoría General de Sistemas y son los siguientes: a) Impulsar el desarrollo de una terminología general que permita describir las características, funciones y comportamientos sistémicos. b) Desarrollar un conjunto de leyes aplicables a todos estos comportamientos $\mathrm{y}$, por 
último, c) Promover una formalización matemática de estas leyes. La Teoría General de Sistemas fue concebida por Ludwig von Bertalanffy en la década de 1940 con el fin de proporcionar un marco teórico y práctico a las ciencias naturales y sociales. (Bertalanffy, 1976).

Después de lo abordado anteriormente, el objetivo de la investigación fue revisar el sistema de indicadores educativos e integrar indicadores para promover la rendición de cuentas en establecimientos públicos de Guatemala.

\section{Método}

El objetivo del estudio fue diseñar un sistema de indicadores educativos para implementar la rendición de cuentas en establecimientos educativos del sector público de Guatemala y para cumplir el mismo se siguió un enfoque mixto, de alcance descriptivo con un diseño no experimental, del tipo transversal.

\section{Participantes}

La población objeto de estudio estuvo integrada por 350 sujetos de 89 establecimientos educativos del sector oficial de 8 municipios de los departamentos de Huehuetenango y San Marcos, para lo cual se aplicó una muestra aleatoria simple. Para los expertos en educación se aplicó una muestra intencionada, mientras que para los directores y técnicos en educación se aplicó el censo. quedando representada de la manera siguiente: para los informantes se realizó un muestreo intencional, aclarando que el mismo no responde a ningún procedimiento estadístico. Los informantes fueron seleccionados por su expertiz en los temas tratados. Se contó con la participación de 5 expertos en educación y programas educativos, 10 técnicos en Educación, 89 directores de los establecimientos mencionados y 184 docentes de nivel primario.

\section{Procedimiento}

Para recopilar información las técnicas seleccionadas fueron la encuesta y el Focus Group. Se aplicó la encuesta a 184 docentes y a 89 directores de establecimientos públicos de los departamentos de Huehuetenango y San Marcos, se realizaron dos grupos focales, uno con cinco expertos en temas de monitoreo y educación y el otro con 10 técnicos, que realizaban acompañamiento educativo en las aulas de los establecimientos mencionados.

\section{Resultados}

De acuerdo a las opiniones de los participantes, las estrategias de rendición de cuentas implementadas son básicas que pueden y deben mejorarse, por experiencia propia en la observación de los participantes se pudo evidenciar que el personal técnico de dependencias del Ministerio de Educación y de algunas Direcciones Departamentales, conocen las estrategias y la forma de aplicarse y como medir los indicadores educativos para tener el nivel de logro o no logro, esto no sucede con los directores y docentes quienes toman la rendición de cuentas como el uso y manejo adecuado de un mural de transparencia y la realización de asambleas periódicas con los padres de familia para informar sobre los gastos y liquidaciones de programas y entrega de calificaciones de sus hijos e hijas. 
Una debilidad detectada es que las estrategias de rendición de cuentas no se comunican de forma adecuada, uno de los especialistas indicó que las estrategias son de una vía. Los logros y alcances de los indicadores educativos pueden mejorarse, si los directores y docentes son informados de los usos que se le dará a la información que generan desde los establecimientos. Además, es muy importante que se indique a que indicador educativo responden y como ellos pueden contribuir al alcance de los mismos, si presentan los datos e información solicitada en los tiempos previstos y de manera objetiva.

Tomando en cuenta la opinión de los especialistas y técnicos en educación se puede inferir que, al contar con el sistema de indicadores educativos, el desafío es lograr que estos sean accesibles para todos los directores de establecimientos públicos, debiendo conocer la forma correcta de darles seguimiento y la temporalidad de cada uno. Quedaría como una tarea para las autoridades educativas locales y directores la correcta socialización de la información a manera de involucrar a los docentes y padres de familia en la implementación de estrategias de rendición de cuentas en cada establecimiento educativo.

Es importante la participación oportuna del personal docente, pero para ello se requiere que tengan competencias tecnológicas básicas. Se debe establecer la mejor forma de capacitar e involucrar a los directores y docentes para generar información confiable y oportuna y entregarla de forma sistemática, además de establecer la estrategia para que sea de fácil acceso cuando este sistematizada en el portal del Ministerio de Educación o de otros medios.

Uno de los informantes indicó acertadamente "Me parece que la rendición de cuentas debería ser una conversación de doble vía donde las comunidades sepan que información está disponible y tener recursos para interpretarla y analizarla. Sólo mostrar las cifras no es suficiente ni garantiza que padres con baja escolaridad entiendan lo que esto significa". En este aspecto se hace necesario realizar reuniones y asambleas comunitarias con la participación de la mayor parte de la comunidad educativa, buscando los mecanismos para que todos comprendan la información que se presenta.

De acuerdo con los resultados obtenidos se hace necesario diseñar un sistema de indicadores que además de ser funcional para usos técnicos de las dependencias del Ministerio de Educación integre a la comunidad educativa en la generación de información que permita mejorar la gestión desde los establecimientos involucrando a las comunidades educativas contribuyendo a mejorar la calidad de la educación que se ofrece.

El diseño requiere potenciar los indicadores con los que ya se cuenta por medio de una campaña de socialización de los mismos y sus resultados a los directores, docentes y comunidad educativa. Lo más importante integrar al diseño actual otra dimensión que incluya indicadores de rendición de cuentas, la información para construir estos indicadores debe de ser reportada desde los establecimientos educativos con participación de directores, docentes, padres de familia y autoridades educativas municipales.

El diseño teórico de este sistema de indicadores quedaría tal como se presenta en la Tabla 1 a continuación: 
Tabla 1

Dimensión indicadores de rendición de cuentas

\begin{tabular}{|c|c|c|c|}
\hline & Indicador & Descripción & Forma de Medición \\
\hline 1. & $\begin{array}{l}\text { Uso del Curriculum } \\
\text { Nacional Base CNB }\end{array}$ & $\begin{array}{l}\text { Docentes formados adecuadamente, } \\
\text { impartiendo sus clases con el } \\
\text { Currículo Nacional Base. }\end{array}$ & $\begin{array}{l}\text { 1.1 Capacitaciones recibidas. } \\
\text { 1.2 Revisión de planificaciones. } \\
\text { 1.3 Acceso al Curriculum Nacional } \\
\text { Base CNB }\end{array}$ \\
\hline 2. & 180 días de clases & $\begin{array}{l}\text { Maestros y maestras que impartan, } \\
\text { por lo menos, } 180 \text { días de clase al } \\
\text { año para desarrollar las } \\
\text { competencias en sus estudiantes. }\end{array}$ & $\begin{array}{l}2.1 \text { Reporte de día de clases } \\
\text { efectivos reportados en el libro de } \\
\text { asistencia de docentes. }\end{array}$ \\
\hline 3. & Materiales de texto & $\begin{array}{l}\text { Un libro de texto por área curricular } \\
\text { y con una bolsa escolar para cada } \\
\text { niño y niña }\end{array}$ & $\begin{array}{l}\text { 3.1 Reporte de entrega de libros de } \\
\text { texto primer trimestre del ciclo } \\
\text { escolar. } \\
\text { 3.2 Reporte de inventario de libros. }\end{array}$ \\
\hline 4. & $\begin{array}{l}\text { Horas efectivas de } \\
\text { clases }\end{array}$ & $\begin{array}{l}\text { Que los estudiantes inviertan } 810 \\
\text { horas anuales para aprender por si } \\
\text { mismos ( } 4.5 \text { horas diarias por } 180 \\
\text { días de clase). }\end{array}$ & 4.1 Agenda de clases \\
\hline 5. & $\begin{array}{l}\text { Un docente por cada } \\
25 \text { niños. }\end{array}$ & Un docente por cada 25 niños. & $\begin{array}{l}5.1 \text { Inventario de docentes por } \\
\text { establecimiento comparado con la } \\
\text { cantidad de alumnos inscritos. }\end{array}$ \\
\hline 6. & $\begin{array}{l}\text { Infraestructura } \\
\text { adecuada }\end{array}$ & Aulas y escritorios adecuados. & $\begin{array}{l}6.1 \text { Reporte de inventario e } \\
\text { infraestructura. }\end{array}$ \\
\hline 7. & Servicios básicos & $\begin{array}{l}\text { Escuelas con servicios básicos, } \\
\text { electricidad, sanitarios funcionales, } \\
\text { agua. }\end{array}$ & 7.1 Observación \\
\hline 8. & $\begin{array}{l}\text { Participación de } \\
\text { padres de familia. }\end{array}$ & $\begin{array}{l}\text { Padres de familia integrando la } \\
\text { organización de padres, participando } \\
\text { en escuela de padres y gestión del } \\
\text { establecimiento. }\end{array}$ & $\begin{array}{l}8.1 \text { porcentaje de padres que } \\
\text { participan }\end{array}$ \\
\hline
\end{tabular}

Nota: Fuente: elaboración propia

El sistema de indicadores actual presenta cinco dimensiones. En la siguiente tabla se presentan las dimensiones como quedarían al integrar al actual la dimensión 6 con una breve descripción de la dimensión y la cantidad de indicadores que incluye.

Tabla 2

Dimensiones del sistema de indicadores educativos

\section{Indicadores}

1. Indicadores de contexto

Esta dimensión incluye cinco indicadores que miden aspectos generales de la población estudiantil.

\section{Cantida}

d

5 Se relaciona al uso de recursos asignados a los 
establecimientos incluyendo los servicios de apoyo.

3. Indicadores de procesos Incluye índices de procesos internos que se educativos

miden en visitas aleatorias a las escuelas.

4. Indicadores de Resultados En esta dimensión se miden las tasas de de Escolarización y promoción, retención y fracaso escolar.

Eficiencia Interna

5. Indicadores de resultados Miden el nivel de logro en Lectura y matemática de aprendizaje de primaria, tercero básico y graduandos.

6. Indicadores de rendición de Estos indicadores se relacionan a procesos de cuentas (propuesta) gestión, que deben ser gestionados desde el

Nota: Fuente: Elaboración propia

\section{Desafíos de construir indicadores educativos}

Para construir indicadores educativos se identificaron los principales desafíos siendo entre otros los que se listan a continuación: a) las competencias tecnológicas de directores y docentes, b) Conocimientos sobre la importancia de la rendición de cuentas, c) Información de retorno a los establecimientos sobre logros y aspectos a mejorar, d) la actitud de los docentes, e) resistencia al cambio.

Para la construcción de indicadores se hace necesario establecer las competencias tecnológicas de los directores y docentes, pues son ellos quienes deben tener ciertas destrezas y habilidades que les permitan realizar los informes y reportes que alimentan las bases de datos del Ministerio de Educación que son gestionadas a través de la Dirección General de Planificación -DIPLAN-. En ese sentido se consultó sobre el nivel de uso de tecnología que se integra con el uso de celular, computadoras, laptops, tabletas y cañonera, para tener la seguridad de que cuando se requiere los docentes pueden utilizar tecnología de forma adecuada para reportar, es interesante observar que el $74 \%$ indicaron tener un buen uso de estos dispositivos.

Además, se consultó sobre el uso del paquete office, que incluye Word en este caso para elaborar informes, Excel, para manejar bases de datos y tablas con información que se les solicita y Power Point para presentar información en diferentes reuniones. En la mayoría de veces tanto docentes como directores deben realizar reportes y presentarlos. Un $81.3 \%$ de encuestados indican tener buen uso de este paquete. Con relación al uso de redes sociales, correo electrónico, Facebook, WhatsApp el $77 \%$ indicaron tener bueno y muy buen uso de las mismas esto es buen indicador de que pueden mejorar la calidad y rapidez en la entrega de información. 


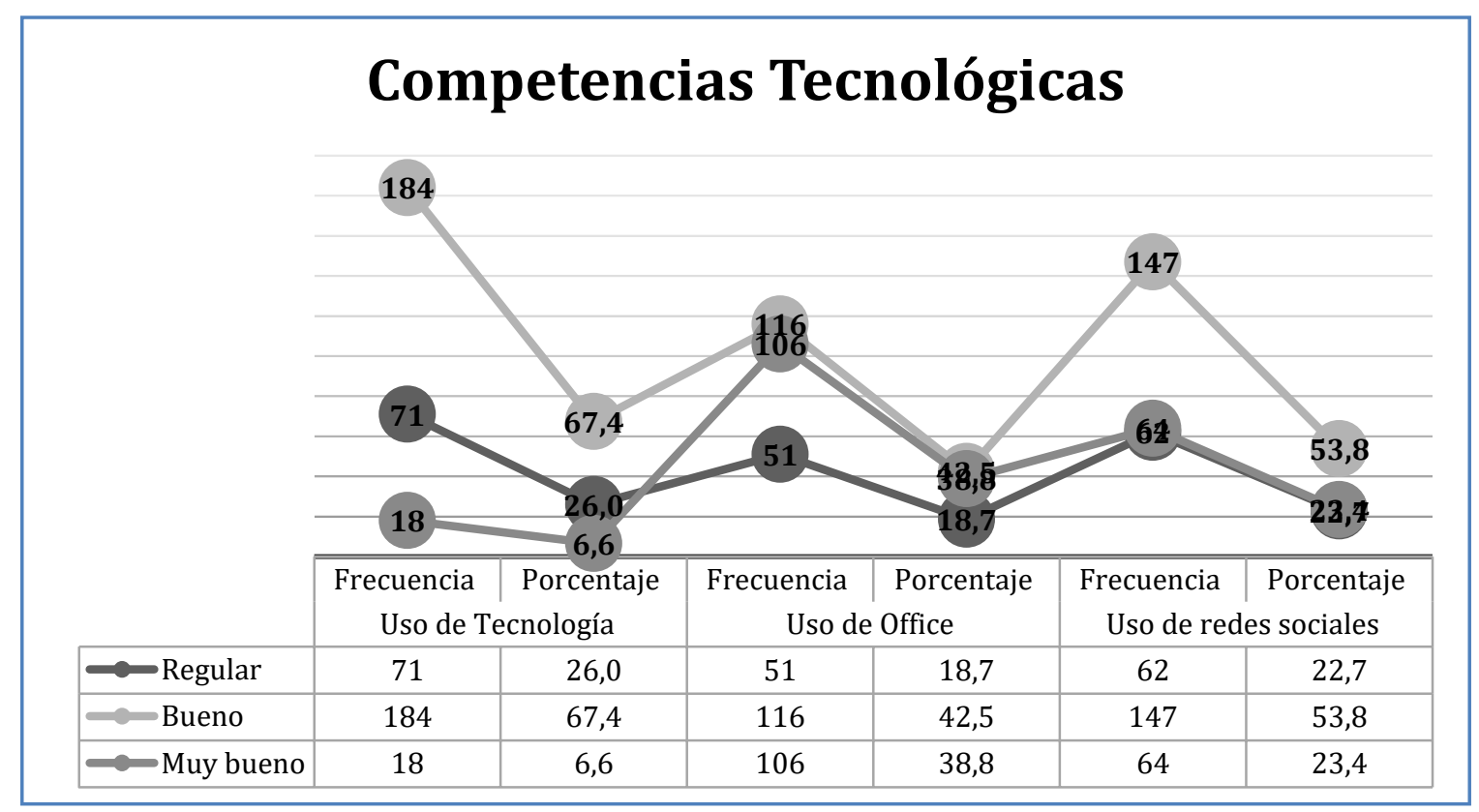

Figura 1 Desafíos para construir indicadores

Nota: Fuente: Elaboración propia

Los informantes expresan que no se entiende con claridad por qué se debe rendir cuentas, los beneficios que esta acción puede implicar para el establecimiento y para el sistema educativo en general. Otro de los desafíos que se evidencia es que los directores y docentes hacen entrega de la información que se les solicita, nunca tienen información de retorno que les clarifique cuáles son sus resultados, como pueden ubicar su nivel de logro y comparar sus tendencias entre grados, entre años, entre establecimientos.

Al ser desconocidos para los docentes los términos, indicador educativo y rendición de cuentas, la actitud de ellos, es un desafío grande al integrar un sistema de indicadores participativo, teniendo que romper primero la resistencia al cambio, informar sobre estos temas, sus fortalezas y debilidades, así como las ventajas que implica contar con indicadores para monitorear los avances.

A la luz de la opinión de los informantes es muy importante realizar un proceso participativo de rendición de cuentas, en donde las bases que, en este caso, serían los directores, docentes, padres de familia y autoridades locales, deben involucrarse activamente en brindar la información en un tiempo oportuno para alimentar las bases de datos que generan los indicadores educativos.

Otro desafío mencionado es la resistencia al cambio que presentan los docentes, indicado como la actitud que se tiene ante la rendición de cuentas. Se ha evidenciado por medio de observación que los docentes se resisten a proporcionar información en los tiempos oportunos debido a varias razones, mencionando entre otras las que se listan: a) Falta de competencias tecnológicas. b) Falta de conectividad en los establecimientos del área rural. c) El costo de contar con red de internet para enviar la información. d) Poco uso del paquete de Office.

En el diagnóstico situacional de las estrategias de rendición de cuentas que el Ministerio de Educación ha implementado se identifican tres ejes: la generación de 
información, el reporte de indicadores y la rendición de cuentas. Cada uno incluye actividades que los directores y docentes realizan en diferentes espacios durante el ciclo escolar.

- $\quad$ La generación de información

- Que incluye a) compartir información, b) utilizar el sistema de registros educativos SIRE, c) presentación de los cuadros finales PRIM, d) generar certificados, e) generar diplomas, f) consultar el curriculum nacional base en línea, g) completar y descargar formularios, e) realizar consultas en el portal del Ministerio de Educación.

- $\quad$ En la figura 2 se visualiza que el mayor porcentaje de encuestados (56.4\%) tienen un uso muy bueno de estas formas de reportes. Eso indica que si podrían generar información para el sistema de indicadores.

- $\quad$ Reporte de indicadores

- Este eje incluye a) conteo rápido, b) ingreso de códigos de estudiantes, c) reporte de liquidaciones, e) reporte de estadística inicial y final. Es en este eje en donde se evidencia que el $78 \%$ de los encuestados saben cómo y cuándo realizar los reportes respectivos.

- $\quad$ Rendición de cuentas

- Incluye a) informes, b) asambleas, c) liquidaciones, e) participación en la comisión de Finanzas, f) integrar la Junta escolar, g) uso adecuado del mural de transparencia, h) presentación de estados financieros a la comunidad educativa, i) planificaciones de compras. En la figura 2 se aprecia que el $47.3 \%$ de encuestados participan en todas las actividades descritas, pero es necesario mejorar para involucrar de forma asertiva al $100 \%$.

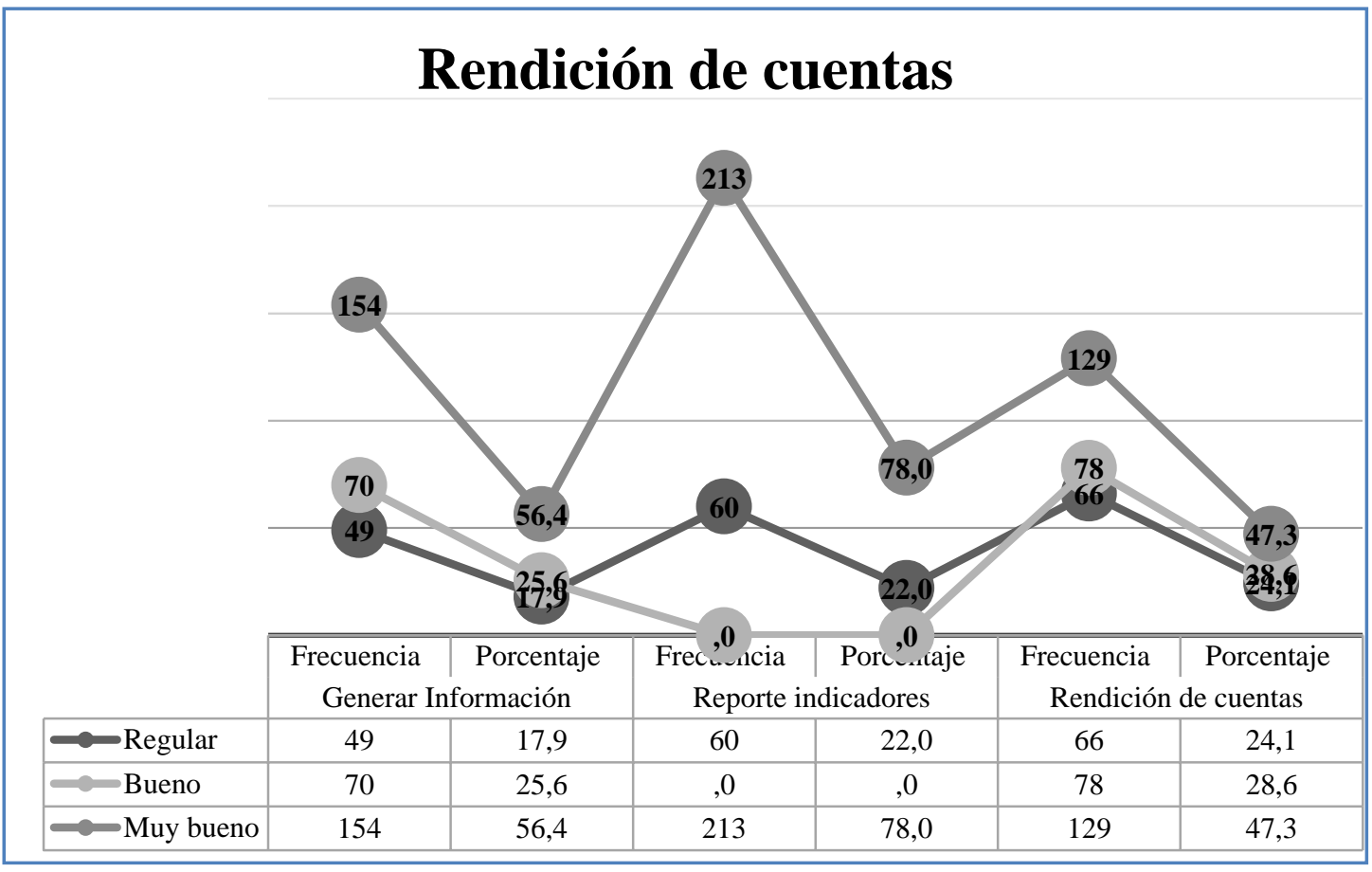

Figura 2. Rendición de cuentas

Nota: Fuente: Elaboración propia 
Los directores y docentes de los diferentes establecimientos toman como otra forma de rendición de cuentas el reporte de recepción, ejecución y liquidación de fondos para los programas de apoyo, alimentación escolar, valija didáctica y útiles escolares, siendo la alimentación escolar la que les demanda más tiempo por ser entre tres a cuatro desembolsos los que reciben en el año. Además, de que para ejecutar este programa se requiere de tener organizadas y legalizadas la organización de padres de familia, comisión de refacción conformada por docentes y padres, comisión de finanzas.

Se diseñó un Mural de Transparencia con la iniciativa de una organización llamada Empresarios por la Educación. Éste mural incluye de manera gráfica información sobre, cantidad de docentes, cantidad de estudiantes, desembolsos recibidos, cantidad recibida, cantidad ejecutada. La instrucción girada es que éste se actualice constantemente y que se coloque en un lugar visible para que la comunidad en general esté informada sobre aspectos relacionados con el personal docente, desembolsos recibidos $\mathrm{y}$ desembolsos ejecutados. No todos los establecimientos cuentan con este mural.

En la encuesta aplicada a directores y docentes de los establecimientos se les preguntó sobre las acciones de rendición de cuentas que realizan. El 73\% indicaron realizar siempre asambleas comunitarias y actualizar el mural de trasparencia. Además, le sigue en el orden realizar asambleas de rendición de cuentas que son las reuniones con padres de familia para presentar la ejecución de los fondos recibidos. En la tabla 3 se detalla la información relacionada a las acciones que realizan en los establecimientos educativos y que para ellos representa rendición de cuentas.

Tabla 3

Acciones de rendición de cuentas

\begin{tabular}{lcccccc}
\hline \multicolumn{1}{c}{ Actividades } & \multicolumn{5}{c}{ Frecuencia de reporte } \\
& \multicolumn{7}{c}{ nunca } & raras veces & siempre \\
\hline Presentar informes financieros & 107 & 9.2 & 38 & 3.9 & 28 & 6.9 \\
Asambleas comunitarias & 31 & 1.4 & 41 & 5.0 & 01 & 3.6 \\
Entrega de liquidaciones & 96 & 5.2 & 41 & 5.0 & 36 & 9.8 \\
Integrar comisión de finanzas & 128 & 6.9 & 66 & 4.2 & 9 & 8.9 \\
Integrar organización de padres & 166 & 0.8 & 45 & 6.5 & 2 & 2.7 \\
Actualizar mural de transparencia & 55 & 0.1 & 17 & .2 & 01 & 3.6 \\
Asamblea para rendir cuentas & 53 & 9.4 & 26 & .5 & 94 & 1.1 \\
Liquidación programas de apoyo & 71 & 6.0 & 33 & 2.1 & 69 & 1.9 \\
Presentar estados financieros & 89 & 2.6 & 41 & 5.0 & 43 & 2.4 \\
Planificar compras alimentación escolar & 69 & 5.3 & 38 & 3.9 & 66 & 0.8 \\
Informar a los padres de familia & 32 & 1.7 & 70 & 5.7 & 71 & 2.6 \\
\hline
\end{tabular}


Nota: Fuente: Elaboración propia, base de datos encuesta (2017)

El manejo adecuado de los recursos financieros que se destinan para fondo de gratuidad, valija didáctica para docentes, útiles escolares y alimentación escolar ha sido una gran preocupación, se han realizado intentos por integrar a los padres de familia para que sean los responsables directos de velar por la buena ejecución, no en todos los casos se ha logrado.

Se preguntó a directores y docentes ¿quienes realizan la recepción de fondos? el mayor porcentaje (89\%) respondieron que la organización de padres de familia; un $11 \%$, indicaron que el director, el otro $11 \%$ mencionaron que se encargaba la comisión de finanzas. Estos son aspectos que requieren de un estudio más profundo.

Por observación participante y visita a los establecimientos se pudo establecer que tanto directores como docentes desconocen la importancia de la información que generan, como matrícula inicial y final, cuadros con información de alumnos promovidos, no promovidos, retirados; para generar los indicadores de procesos educativos.

En talleres didácticos con directores y docentes se realizaron prácticas de ingreso al portal del Ministerio de Educación, para orientarlos sobre su uso correcto en la toma de información. La mayoría desconocía la forma de ingresar y la información que se publica, coincidiendo con la opinión de algunos entrevistados que indicaron que se cuenta con un sistema de indicadores relativamente muy bueno pero que los actores de base que son directores, docentes y padres de familia no lo conocen.

\section{Ventajas que se presentan en el diseño de un sistema de indicadores}

Según la opinión de los participantes en los grupos focales las ventajas de contar con un sistema de indicadores que promueva una eficiente rendición de cuentas son las que se listan:

- Disminución de la corrupción en el manejo y uso de fondos en los establecimientos educativos, además de generación de un reporte confiable de matrícula escolar y personal docente en los establecimientos.

- Cómo práctica internacional, la ventaja está en promover la confianza en el sistema educativo nacional, generando con ello más acciones de apoyo.

- Con la información obtenida se pueden generar estrategias de mejora durante el proceso que pueden incidir en lograr la mejora de la calidad de la enseñanza aprendizaje.

- Se pueden medir la evolución del proceso educativo, logros, avances y/o retrocesos, tomando las medidas correctivas si fuera necesario.

- La participación de los padres de familia y de los docentes de manera responsable, construyendo de esa forma una ciudadanía responsable y participativa.

- Realizar comparaciones nacionales e internacionales, permitiendo con ello formar parte de los países que se han integrado a diferentes acciones y estrategias para alcanzar los objetivos del milenio y los objetivos de Educación para Todos.

Desventajas en el diseño de un sistema de indicadores educativos para promover la rendición de cuentas. 
Una de las desventajas que se identifica es la resistencia al cambio que presenta el gremio magisterial. Según la opinión de los especialistas en educación entrevistados, el sistema de indicadores no presenta desventajas per se. Las desventajas se visualizan por la poca promoción que se hace de la forma en que funciona, los datos que manejan, la forma en que recopilan información, los resultados obtenidos por establecimiento, por municipio y por departamento.

La aplicación de evaluaciones estandarizadas de forma muestral es otra de las desventajas identificadas, debido a que los establecimientos que conforman el marco muestral tienen poco conocimiento sobre el uso de esa información, además de que no se les comparte de forma amigable los resultados obtenido. En el caso de establecimientos que no forman esa muestra desconocen que existen esas evaluaciones.

Los indicadores existen, pero fueron construidos por expertos, no se contó con la participación de los docentes, es una debilidad porque ellos no se sienten parte del proceso, desconocen la forma en que se utiliza la información, siendo ellos los que proporcionan la información que alimentan las bases de datos de donde se obtienen los indicadores educativos.

\section{Validación del sistema de indicadores}

En opinión de los entrevistados el proceso de rendición de cuentas es incipiente, además, hay muchos retos que deben superarse, especialmente el rechazo al cambio que presentan los docentes cuando se implementan acciones desconocidas, específicamente si son medidas de control. Por lo tanto, se requiere de sensibilización para que se de ese cambio de cultura.

Por medio de entrevistas y de observación participante se pudo evidenciar que se realizan acciones de monitoreo como una forma de rendir cuentas, sin embargo, estas sólo permiten observar situaciones aisladas, que no genera información confiable y oportuna para tomar decisiones remediales, a los directores y docentes no se les da información de retorno que les permita saber cuáles son sus logros y cuáles son sus debilidades.

Un aspecto que debe fortalecerse para que se dé una adecuada rendición de cuentas, es la incorporación de los padres de familia en los procesos de gestión. Se han hecho esfuerzos por integrarlos, pero en algunos casos son excluidos a conveniencia del personal docente de los establecimientos. Se deben implementar procesos de comunicación de doble vía que permitan generar un mejor flujo de información desde las bases o sea los docentes.

Otro informante clave opina lo siguiente "Me parece que la rendición de cuentas debería ser una conversación de doble vía dónde las comunidades sepan que información está disponible y tener recursos para interpretarla y analizarla. Solo mostrar las cifras no es suficiente ni garantiza que padres con baja escolaridad entiendan lo que esto significa".

Otra de las opiniones compartida es que el sistema educativo requiere de un monitoreo constante, que permita generar información, a la que debe dársele seguimiento y atender las recomendaciones que se den. De las limitantes que se identificaron se puede mencionar que en los establecimientos no se recibe información sobre los niveles de logro o no logro, del estado de las tasas de promoción, retención, deserción y se carece de una cultura de análisis de los datos de la escuela. En la mayoría de las ocasiones el director y los docentes, no tienen acceso a internet o a la pizarra 
digital en donde se presentan los diferentes indicadores y las estadísticas, provocando no ser accesibles para analizar estrategias de mejora a implementar.

\section{Discusión y conclusiones}

Los resultados de este estudio permitieron evidenciar que los docentes y directores encuestados tienen pocas competencias tecnológicas, es importante mencionar que estas deben mejorarse por medio de actualización. En relación al uso del paquete Office un $46.9 \%$ indicó utilizar siempre Word, el $12.1 \%$ respectivamente indicaron utilizar Excel y Power Point. Respecto al sistema de registros educativos SIRE- $36.6 \%$ indican utilizarlo este es muy importante porque permite el ingreso de datos de los estudiantes de todos los niveles.

El poco uso que se evidencia del programa Excel, puede ser una gran debilidad y el mayor desafío para generar información que alimente las bases de datos del sistema de indicadores, pues la mayoría de información que administran en la Dirección General de Planificación se presenta en tablas de Excel. El 59.4\% respondieron que no utilizan Google, se requiere de utilizarlo adecuadamente porque se facilitaría el acceso al portal del Ministerio de Educación y en especial al sistema de indicadores.

Los resultados cualitativos de este estudio permitieron evidenciar que existe en Guatemala un sistema de indicadores educativos, diseñado en el Ministerio de Educación, que no incluye indicadores de rendición de cuentas que permitan integrar a la comunidad educativa en el fomento de la transparencia. Tanto directores, docentes, estudiantes y padres de familia deben contar con competencias tecnológicas para participar activamente en el proceso de rendir cuentas.

El diseño de un sistema de indicadores que incluya en su estructura una dimensión relacionada a la rendición de cuentas influirá en la gestión administrativa de establecimientos públicos, con este agregado se tendrán mayores posibilidades de encontrar establecimientos con mejores prácticas de accountability, ya que se ha evidenciado de forma empírica que la integración de la comunidad educativa de forma organizada contribuye en que los resultados administrativos y de logro de los estudiantes mejoren notablemente.

Dentro de los desafíos que surgen cuando se construyen indicadores educativos, el mayor es, el de lograr la integración de la comunidad educativa, tanto en la construcción como en la generación de información válida, confiable y oportuna para rendir cuentas. Previo a esta construcción todos los actores deben tener la claridad de que es la Rendición de cuentas y cuáles son sus alcances.

Al construir un sistema de indicadores se presenta el desafío de contar con la forma técnica de recopilar y administrar la información, con la tecnología adecuada y personas capacitadas en el manejo de bases de datos, de manera que esta información pueda ser procesada en el menor tiempo posible y socializada con todos los implicados en el proceso educativo.

Se debe de actualizar a la comunidad educativa en temas de transparencia y vencer la resistencia al cambio. Esto resulta una tarea difícil ya que los docentes tienen, como todos los humanos, mucha resistencia a implementar cambios, Se debe incluir en 
los programas de actualización a todos los docentes sin distinción por nivel educativo, atendiendo que los docentes del renglón 021. en algún momento, pueden formar parte del renglón 011 .

Otro desafío que hay que enfrentar con suma urgencia, es el de implementar de forma técnica la entrega de información por parte del Ministerio de Educación hacia los docentes. Siempre se piden datos, sin explicación del uso de los mismos ni de los resultados que se esperan, por lo que se debe de brindar a todos los establecimientos un informe de información de retorno, en donde se visualice el uso de la información, los resultados, los logros y avances y los aspectos en que deben y pueden mejorar. De esa manera a nivel de establecimiento se puede realizar comparaciones durante el año, entre años y entre establecimientos como acciones de rendición de cuentas y de mejora continua.

Al realizar un diagnóstico situacional de las estrategias de rendición de cuentas que el Ministerio de Educación ha implementado, se logró evidenciar que estas son incipientes, que se deben integrar de mejor forma a la comunidad educativa para que se sumen a los esfuerzos por lograr la mejora de la calidad.

Cuando se promueve la rendición de cuentas de forma correcta y de manera responsable, se logra la integración de la comunidad educativa. De esa manera se dan responsabilidades compartidas que pueden generar mejoras en todo el proceso educativo. Se ha evidenciado que, con una mejor participación de los padres de familia, el nivel de eficiencia de los establecimientos mejora.

Implementar rendición de cuentas es un tema que cuando no se conoce provoca reacciones diversas. En este caso, los docentes que no han sido informados, presentan resistencia a lo desconocido. Por ello se deben de realizar constantemente reuniones informativas para que se tenga una mejor visión de la importancia de la rendición de cuentas y cómo lograrla al implementar un sistema de indicadores.

\section{Referencias}

Arnold, M., \& Rodríguez , D. (1998). Sociedad y teoría de sistemas: elementos para la comprensión de la teoría de Niklas Luhman. Universidad de Chile: Editorial Universitaria. Retrieved from http://www.moebio.uchile.cl/03/frprinci.htm.

Banco Mundial . (2004). World Development Report (WDR). Colombia : Panamericana Formas.Retrieved from /www.openknowledge.worldbank.org.

Bertalanffy, L. (1976). Teoría General de los Sistemas .México: Fondo de Cultura Económica de México.

Corvalán, J.(2006). Accountability educacional: Rendición de cuentas más responsabilización por los procesos y resultados de la educación. Chile: San Marino.

García , M., Raez, L., Castro, M., Vivar, L., \& Oyola, L. (2003). Sistema de indicadores de calidad. Industrial Data, 6 (2), 63-65. doi 10.15381/idata.v6i2.6078.

Hernández Sampieri, R., Fernández , C., \& Baptista , P. (2010). Metodología de la Investigación. México: MacGraw Hill. 
Manno, B., McMeekin, R., Puryear, J., Winkler, D., \& Winters, M. (2006). Accountability educacional:posibilidades y desafíos para América Latina a partir de la experiencia internacional. Santiago de Chile: CIDE. Retrieved from http://repositorio.minedu.gob.pe/handle/123456789/4432.

Martínez , F. (2010). Los indicadores como herramientas para la evaluación de la calidad de los sistemas educativos. Sinéctica , 19-38. doi S1665109X2010000200004.

Martínez , J. (2010). La construcción de indicadores y evaluación de calidad en centros educativos de México. REICE, 133-153. Retrieved from http://www.redalyc.org/articulo.oa?id=55119084010

McMeekin, R. (2006). Acreditación, Accountability y Mejoramiento de la Calidad de la Educación en Escuelas Vulnerables. Pensamiento Educativo, 237-253.

Ministerio de Educación.(2013). Sistema Nacional de Indicadores Educativos . Guatemala.

Ministerio de Educación Guatemala. (2016). Memoria de Labores 2015. Guatemala.

Morales, F. (6 de marzo de 2016). Teoría General de sistemas. Retrieved from http://tgsfreddymorales.blogspot.com/

Morduchowicz, A. (2006). Los Indicadores Educativos y las dimensiones que los integran. Buenos Airs, Argentina : UNESCO. Retrieved from http://www.iipebuenosaires.org.ar/.

Programa de promoción de la Reforma Educativa PREAL. (2006). Accountability educacional: posibilidades y desafíos para América Latina a partir de la experiencia internacional. Santigo de Chile. doi 123456789/4432.

Programa de Promoción de la Reforma Educativa en América Latina PREAL. (2001). Quedándonos Atrás: Un informe del progreso educativo en América Latina. Chile. Retrieved from https://www.oei.es/historico/quipu/Informe_preal2001.pdf

Santizo, C. (2006 ). Mejorando la Rendición de Cuentas y la Transparencia a través de la Participación Social: el Programa escuelas de calidad en México. REICE.Revista Iberoamericana sobre Calidad, eficacia y Cambio en Educación, 4 (1),38-51. doi articulo. oa55140105.

Scheerens, J., Glass, C., \& Thomas, S. (2003). Educational Evaluation, Assessment, and monitoring. A Systemic Approach. New York: Taylor \& Francis. doi 9789026519598

USAID/AED Proyecto Diálogo para la Inversión Social en Guatemala. (2006). Sistema Nacional de Indicadores Educativos . Guatemala. 
Fecha de recepción: 02/12/2017

Fecha de revisión: 08/10/2018

Fecha de aceptación: 19/01/2019 